\title{
Aleitamento materno e seu conhecimento por alunos de enfermagem
}

RESUMO | Objetivo: Investigar o conhecimento dos alunos de enfermagem sobre o aleitamento materno. Métodos: Abordagem transversal, qualitativa e quantitativa de campo desenvolvido em Universidade particular do interior de São Paulo. A coleta de dados foi realizada utilizando-se questionário, com 15 perguntas sobre amamentação, distribuídas em três partes. Foram incluídas perguntas sobre manejo na amamentação, aspectos legais na proteção ao aleitamento materno e benefícios da amamentação para o binômio. Analisou-se os dados por meio da descritiva simples e para as comparações foi utilizado a análise de variância. Resultados: Os alunos de enfermagem do oitavo semestre obtiveram desempenho geral de 81,2\% nas respostas corretas, valor estatisticamente superior a uma das turmas do sétimo período, mas semelhante à outra turma. Assim a turma sétimo A e oitavo tiveram resultados significativos na pesquisa. Conclusão: Apesar do bom conhecimento sobre as vantagens do aleitamento, evidenciou-se conhecimento inferior ao esperado no que se refere ao processo fisiológico do aleitamento materno.

Palavras-chaves: Aleitamento materno; Conhecimento; Estudantes de enfermagem.

\begin{abstract}
Objective: To investigate the knowledge of nursing students about breastfeeding. Methods: Cross-sectional, qualitative and quantitative approach developed at a private university in an inner city in São Paulo State. Data collection was performed using a questionnaire comprising 15 questions about breastfeeding, distributed in three parts. Questions about breastfeeding management, legal aspects of breastfeeding protection and breastfeeding benefits were included. Data were analyzed using the simple descriptive and variance analysis was performed for comparisons. Results: Nursing students in the eighth semester achieved an overall performance of $81.2 \%$ in the correct answers, a value statistically superior to one of the classes in the seventh period, but similar to the other. Conclusion: Despite the good knowledge about the advantages of breastfeeding, knowledge was shown to be lower than expected with regard to the physiological process of breastfeeding.
\end{abstract}

Keywords: Breastfeeding; Knowledge; Nursing students

RESUMEN | Objetivo: Investigar el conocimiento de los estudiantes de enfermería sobre la lactancia materna. Métodos: enfoque transversal, cualitativo y cuantitativo desarrollado en una universidad privada en una ciudad del interior del estado de São Paulo. La recolección de datos se realizó mediante un cuestionario que comprende 15 preguntas sobre lactancia materna, distribuidas en tres partes. Se incluyeron preguntas sobre el manejo de la lactancia materna, los aspectos legales de la protección y los beneficios de la lactancia materna. Los datos se analizaron mediante el análisis descriptivo simple y se realizó un análisis de varianza para las comparaciones. Resultados: Los estudiantes de enfermería en el octavo semestre lograron un rendimiento general del $81.2 \%$ en las respuestas correctas, un valor estadísticamente superior a una de las clases en el séptimo período, pero similar al otro. Conclusión: a pesar del buen conocimiento sobre las ventajas de la lactancia materna, se demostró que el conocimiento es menor de lo esperado con respecto al proceso fisiológico de la lactancia materna.

Palavras claves: Lactancia Materna; Conocimiento; Estudiantes de enfermería.

\section{Ana Beatriz Moraes Martins}

Faculdade de Ciências da Saúde/Curso de Enfermagem, Universidade do Oeste Paulista, Presidente Prudente, SP, Brasil. ORCID: 0000-0002-6880-1707

\section{lara Cristina Vieira}

Faculdade de Ciências da Saúde/Curso de Enfermagem, Universidade do Oeste Paulista, Presidente Prudente, SP, Brasil. ORCID: 0000-0003-3183-4211

\section{Jossimara Polettini}

Faculdade de Ciências da Saúde/Curso de Enfermagem, Universidade do Oeste Paulista, Presidente Prudente, SP, Brasil. Universidade Federal da Fronteira Sul/ Campus Passo Fundo, RS, Brasil.

ORCID: 0000-0002-6719-8715
Lucas Lima de Moraes

Faculdade de Ciências da Saúde/Curso de Enfermagem, Universidade do Oeste Paulista, Presidente Prudente, SP, Brasil. ORCID: 0000-0003-1613-5068

\section{Wellington Garcia Siqueira}

Residência em Enfermagem em Saúde da Criança, Universidade Estadual de Londrina, Londrina, PR, Brasil.

ORCID: 0000-0002-6837-3511

\section{Glilciane Morceli}

Faculdade de Ciências da Saúde/Curso de Enfermagem, Universidade do Oeste Paulista, Presidente Prudente, SP, Brasil. Universidade do Estado de Minas Gerais/ UEMG/Unidade Passos, MG, Brasil. ORCID: 0000-0001-8216-9931
INTRODUÇÃO

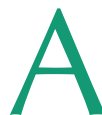
amamentação é a principal fonte de nutrientes ao recém-nascido, assim considerada prática fundamental para o desenvolvimento saudável da criança. $\mathrm{O}$ leite materno é um alimento natural e rico em gorduras, proteínas, imunológicos e demais nutrientes, fornecendo assim a energia vital para o desenvolvimento do recém-nascido. ${ }^{(1,2)}$

O enfermeiro é fundamental na promoção e manejo da amamentação, uma vez que se trata de uma prática em que muitas barreiras podem ser evidenciadas durante este período, ainda, o enfermeiro encontra-se em todos os serviços de serviço atuando desde o pré-natal até o puerpério. ${ }^{(3)}$ É 
essencial que o enfermeiro atue ativamente no processo de amamentação auxiliando a puérpera e orientando-a desde a primeira mamada, esclarecendo dúvidas e orientando as benfeitorias da prática de amamentar, que é considerada milenar pelos seus benefícios amplos para o binômio mãe/ recém-nascido. ${ }^{(4,5)}$

A principal estratégia da Organização Mundial da Saúde (OMS) para reduzir desfechos perinatais desfavoráveis e a mortalidade infantil é a promoção do aleitamento materno em todo o mundo(6), portanto a promoção do aleitamento materno deve ser uma das ações prioritárias nos serviços de saúde. O aleitamento materno contribui no desenvolvimento da criança protegendo contra alergias; doenças crônicas não transmissíveis; promove o desenvolvimento neurológico adequado e estimula o vínculo entre o binômio ${ }^{(7,8)}$.

$\mathrm{O}$ aleitamento materno previne o índice de mortes infantis e ainda promove a saúde física, mental e psíquica da criança. Estima-se que a amamentação reduz $13 \%$ as mortes em crianças menores de 05 anos e de 19 a $22 \%$ as mortes neonatais, se praticada na primeira hora de vida. Estudos mostram que a média de duração do aleitamento materno exclusivo é de 1,8 meses e a média de aleitamento materno com complementação é de 11,2 meses. ${ }^{(9,10)}$

Com o avanço científico e a maior divulgação sobre o aleitamento materno, a prática teve seu aumento no Brasil, principalmente na prevalência do aleitamento materno exclusivo em menores de 06 meses, com taxas de $41 \%$ nas capitais brasileiras e Distrito Federal. ${ }^{(11,12}$

Por esta razão, durante a graduação o acadêmico de enfermagem necessita de informações sólidas, com conhecimento teórico juntamente com a prática, a fim de garantir um cuidado de qualidade, tanto para a mãe quanto para o recém-nascido, com ações de

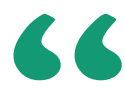

promoção, proteção e apoio ao aleitamento materno. ${ }^{(11,13)}$

Diante do importante papel bio-

Diante do importante papel biológico e cultural do aleitamento materno é necessário que os graduandos de enfermagem compreendam as informações básicas e a prática do aleitamento materno, os conteúdos essenciais sobre aleitamento materno, para assim, fortalecer o aprendizado e colocar em exercício o seu conhecimento adquirido durante o período da graduação. lógico e cultural do aleitamento materno é necessário que os graduandos de enfermagem compreendam as informações básicas e a prática do aleitamento materno, os conteúdos essenciais sobre aleitamento materno, para assim, fortalecer o aprendizado e colocar em exercício o seu conhecimento adquirido durante o período da graduação. $^{(13)}$

Estudos realizados com estudantes de enfermagem, demostraram que o preparo do aluno ainda é falho em relação ao aleitamento materno. Apenas $25 \%$ das enfermeiras indicaram a formação acadêmica como a maior fonte de conhecimento adquirido em aleitamento materno. Outro estudo realizado por uma Universidade Federal, verificou que os universitários, de maneira geral, sentem se inseguros para aconselhar gestantes e/ou lactentes sobre a amamentação, levando sugestões sobre a possível reformulação nos currículos dos cursos, reforçando assim o melhor ensino desta prática nas Universidades. ${ }^{(13)}$

$\mathrm{Na}$ literatura encontram-se apenas alguns estudos que relacionam o conhecimento sobre aleitamento materno entre os e estudantes do curso de graduação em enfermagem, mostrando a fragilidade de discussão dessa associação. ${ }^{(9,13)}$

Frente ao exposto acima, o presente trabalho teve como objetivo avaliar: Qual o conhecimento dos alunos, dos dois últimos períodos do curso de graduação em enfermagem, da Universidade do Oeste Paulista, sobre aleitamento materno?

O estudo caracterizou os aspectos de conhecimento dos alunos sobre aleitamento materno e identificou as áreas nas quais os graduandos apresentaram maior domínio teórico sobre o tema. 
Tabela 1. Caracterização sociodemográfica da população (n= 84).

\begin{tabular}{lcc|}
\hline Variável & $\mathbf{n}$ & $\%$ \\
\hline Sexo & & \\
\hline Feminino & 72 & 85,7 \\
\hline Masculino & 12 & 14,3 \\
\hline Estado civil & & \\
\hline Casado & 24 & 28,6 \\
\hline Solteiro & 60 & 71,4 \\
\hline Número de filhos & & \\
\hline 0 & 64 & 76,2 \\
\hline 1 & 9 & 10,7 \\
\hline 2 & 9 & 10,7 \\
\hline 3 & 2 & 2,4 \\
\hline Já amamentou? & & \\
\hline Não & 67 & 79,8 \\
\hline Sim & 17 & 20,2 \\
\hline Preparo sobre aleitamento materno & & \\
\hline Bem & 30 & 35,7 \\
\hline Mal & 2 & 2,4 \\
\hline Muito bem & 24 & 28,6 \\
\hline Regularmente & 28 & 33,3 \\
\hline
\end{tabular}

Fonte: Os autores/2017

Tabela 2. Porcentagem dos padrões de resposta em relação ao Manejo no AM.

\section{Questões}

0 leite materno é produzido nos alvéolos?

Na hora que a mãe estiver amamentando ela pode sentir dor nos seios?

\begin{tabular}{|c|c|c|c|}
\hline $\begin{array}{l}0 \text { queixo do recém-nascido deve estar tocando o seio } \\
\text { da mãe? }\end{array}$ & 73,8 & 23,8 & 2,4 \\
\hline $\begin{array}{l}\text { Na hora do AM é possível ver as sucções lenta e profun- } \\
\text { da que o RN faz? }\end{array}$ & 97,6 & 1,2 & 1,2 \\
\hline $\begin{array}{l}\text { A boca do RN deve estar bem aberta porem o lábio } \\
\text { inferior deve esta voltada para fora? }\end{array}$ & 84,5 & 9,5 & 6,0 \\
\hline
\end{tabular}

*RN: Recém-nascido *AM: Aleitamento Materno

Fonte: Os autores/2017

Tabela 3. Porcentagem dos padrões de resposta em relação aos aspectos legais na proteção ao AM.

\section{Questões}

O AM tem vantagens nutricionais: adequado no crescimento e desenvolvimento?

O AM tem vantagens imunológicas: proteção contra doenças a curta e longo prazo?
Sim (\%) Não (\%) Não sei (\%)

$9,5 \quad 17,4 \quad 13,1$

$83,3 \quad 16,7 \quad 0,0$

Sim (\%) Não (\%) Não sei (\%)

$97,6 \quad 1,2 \quad 1,2$

$100 \quad 0,0 \quad 0,0$
MÉTODO

Foi realizado um estudo quantitativo, qualitativo e transversal, através da aplicação de questionário contendo as variáveis sociodemográficas e sobre o conhecimento a respeito do aleitamento materno.

Foram incluídos alunos dos dois últimos períodos (semestres) do curso de graduação em enfermagem da Universidade Do Oeste Paulista (UNOESTE) de Presidente Prudente, localizado no interior do Estado de São Paulo. Foram utilizados como critérios de inclusão que os participantes assinassem o Termo de Consentimento Livre e Esclarecido e serem graduando dos dois últimos períodos do curso de Enfermagem da Universidade do Oeste Paulista/ UNOESTE e como critérios de descontinuidade perdas de dados relativos à coleta de dados.

As variáveis de estudo e as características sociodemográficas dos estudantes e os dados pessoais e comportamentais de interesse foram coletados seguindo o questionário estabelecido para o presente estudo.

O estudo foi realizado no período de novembro de 2016 a fevereiro de 2017, e foi previamente aprovado pelo Comitê de Ética em Pesquisa da Instituição CAAE n ${ }^{\circ} 48839815.3 .0000 .5515$. Foram obtidas informações sobre amamentação, cada uma com três possibilidades de resposta: sim, não e não sei. Foram incluídas perguntas sobre manejo na amamentação, aspectos legais na proteção ao aleitamento materno, benefícios da amamentação para a mãe e para o recém-nascido.

Os dados foram armazenados em planilhas do software Excel 2013, conferidos e avaliados em relação à consistência e, posteriormente, submetidos à análise estatística descritiva simples, e, para as comparações dos dados das turmas do curso de enfermagem avaliadas, intra e entre os grupos, foi utilizado o teste de análise de va- 


\begin{tabular}{|c|c|c|c|}
\hline $\begin{array}{l}\text { Pode dar qualquer outro tipo de líquido ou alimento ao } \\
\text { RN além do leite materno? }\end{array}$ & 4,8 & 95,2 & 0,0 \\
\hline Existe duração máxima na amamentação? & 22,6 & 77,4 & 0,0 \\
\hline $\begin{array}{l}\text { O AM tem vantagens psicológicas: aspectos emocionais } \\
\text { para o RN e para a mãe? }\end{array}$ & 98,8 & 0,0 & 1,2 \\
\hline
\end{tabular}

*RN: Recém-nascido *AM: Aleitamento Materno

Fonte: Os autores/2017

Tabela 4. Porcentagem dos padrões de resposta em relação aos Benefícios da

amamentação para a mulher e ao RN.

\begin{tabular}{|c|c|c|c|}
\hline Questões & $\operatorname{Sim}(\%)$ & Não (\%) & Não sei (\%) \\
\hline $\begin{array}{l}\text { O leite materno é difícil de ser digerido e provoca mais } \\
\text { cólica no RN? }\end{array}$ & 1,2 & 95,2 & 1,2 \\
\hline $\begin{array}{l}\text { O leite materno colabora na formação do sistema } \\
\text { imunológico da criança? }\end{array}$ & 97,6 & 0,0 & 2,4 \\
\hline $\begin{array}{l}\text { Na hora da amamentação aumenta o vínculo entre mãe } \\
\text { e filho? }\end{array}$ & 100 & 0,0 & 0,0 \\
\hline 0 leite materno previne anemia? & 71,4 & 13,1 & 15,5 \\
\hline $\begin{array}{l}\text { A amamentação pode proteger a mãe contra o cân- } \\
\text { cer de mama e de colo de ovário? }\end{array}$ & 64,3 & 20,2 & 15,5 \\
\hline \multicolumn{4}{|l|}{ RN: Recém-nascido. } \\
\hline Fonte: Os autores/2017 & & & \\
\hline
\end{tabular}

Tabela 5. Conteúdo das questões e percentual de acertos/questão e média e desvio padrão (dp).

\begin{tabular}{|c|c|c|}
\hline Conteúdo das Questões & Acertos N (\%) & Média \pm DP \\
\hline O leite materno é produzido nos alvéolos? & $8(9,5)$ & $0,56( \pm 0,17)$ \\
\hline $\begin{array}{l}\text { Na hora que a mãe estiver amamentando ela pode } \\
\text { sentir dor nos seios? }\end{array}$ & $14(16,7)$ & $0,56( \pm 0,17)$ \\
\hline $\begin{array}{l}0 \text { queixo do recém-nascido deve estar tocando o seio } \\
\text { da mãe? }\end{array}$ & $62(73,8)$ & $0,56( \pm 0,17)$ \\
\hline $\begin{array}{l}\text { Na hora da amamentação é possível ver as sucções } \\
\text { lenta e profunda que o RN faz? }\end{array}$ & $82(97,6)$ & $0,56( \pm 0,17)$ \\
\hline $\begin{array}{l}\text { A boca do RN deve estar bem aberta porem o lábio } \\
\text { inferior deve estar voltado para fora? }\end{array}$ & $71(84,5)$ & $0,56( \pm 0,17)$ \\
\hline $\begin{array}{l}\text { O AM tem vantagens nutricionais: adequado no } \\
\text { crescimento e desenvolvimento? }\end{array}$ & $82(97,6)$ & $0,94( \pm 0,11)$ \\
\hline $\begin{array}{l}\text { O AM tem vantagens imunológicas: proteção contra } \\
\text { doenças a curta e longo prazo? }\end{array}$ & $84(100)$ & $0,94( \pm 0,11)$ \\
\hline $\begin{array}{l}\text { Pode dar qualquer outro tipo de líquido ou alimento } \\
\text { ao recém-nascido além do leite materno? }\end{array}$ & $80(95,2)$ & $0,94( \pm 0,11)$ \\
\hline Existe duração máxima na amamentação? & $65(77,4)$ & $0,94( \pm 0,11)$ \\
\hline $\begin{array}{l}\text { O AM tem vantagens psicológicas: aspectos emocio- } \\
\text { nais para o RN e para a mãe? }\end{array}$ & $83(98,8)$ & $0,94( \pm 0,11)$ \\
\hline $\begin{array}{l}0 \text { leite materno é difícil de ser digerido e provoca } \\
\text { mais cólica no RN? }\end{array}$ & $82(97,6)$ & $0,86( \pm 0,16)$ \\
\hline $\begin{array}{l}0 \text { leite materno colabora na formação do sistema } \\
\text { imunológico da criança? }\end{array}$ & $82(97,6)$ & $0,86( \pm 0,16)$ \\
\hline
\end{tabular}

riância (ANOVA); para todos os testes realizados, o limite de significância estatística foi de p menor ou igual a 0,05.

\section{RESULTADOS}

Participaram deste estudo 84 alunos do curso de graduação de Enfermagem da Universidade do Oeste Paulista, onde $n=30(35,7 \%)$ do sétimo período A, $n=27(32,15 \%)$ do sétimo período C, e $n=27(32,15 \%)$ do oitavo período do curso. A Tabela 1 mostra a caracterização sociodemográfica dos participantes da pesquisa. A maioria dos participantes era do sexo feminino e disseram que não amamentaram; a maioria dos participantes também declarou ser solteiro(a) e não ter nenhum filho.

A porcentagem dos padrões de resposta em relação ao Manejo no aleitamento materno (AM) estão apresentados na Tabela 2, e observa-se que elevada porcentagem $(13,1 \%)$ dos alunos não souberam responder à questão "O leite materno é produzido nos alvéolos?". A Tabela 3 mostra os aspectos legais na proteção ao aleitamento materno. Todos os alunos responderam corretamente à pergunta "O AM tem vantagens imunológicas: proteção contra doenças a curta e longo prazo" .

Na Tabela 4 foram apresentadas as porcentagens dos padrões de resposta em relação aos benefícios da amamentação para a mulher e ao recém-nascido. Da mesma forma que descrito anteriormente, $100 \%$ dos participantes responderam sim para questão "Na hora da amamentação aumenta o vínculo entre mãe e filho?". por outro lado, 15,5\% dos alunos não souberam responder às perguntas: "A amamentação pode proteger a mãe contra o câncer de mama e de colo de ovário? " e "O leite materno pode prevenir anemia?".

O conteúdo das questões e percentual de acertos por questão e média e desvio estão descritos na Tabela 5. O 


\begin{tabular}{|c|c|c|}
\hline $\begin{array}{l}\text { Na hora da amamentação aumenta o vínculo entre } \\
\text { mãe e filho? }\end{array}$ & $84(100)$ & $0,86( \pm 0,16)$ \\
\hline 0 leite materno previne anemia? & $60(71,4)$ & $0,86( \pm 0,16)$ \\
\hline $\begin{array}{l}\text { A amamentação pode proteger a mãe contra o cân- } \\
\text { cer de mama e de colo de ovário? }\end{array}$ & $54(64,3)$ & $0,86( \pm 0,16)$ \\
\hline
\end{tabular}

Tabela 6. Apresentação do desempenho de cada turma em cada seção do questionário

\begin{tabular}{lcccc} 
Taxa de Acerto & Sétimo & Sétimo & Oitavo \% & p \\
Geral & A \% & C \% & & 0,149 \\
\hline Manejo na Amamentação. & 78,7 & 76,5 & 81,2 & 0,608 \\
\hline Aspectos legais na proteção ao AM. & 54,0 & 57,0 & 58,5 & 0,608 \\
\hline $\begin{array}{l}\text { Benefícios da amamentação para a mulher } \\
\text { e ao RN. }\end{array}$ & 97,3 & 91,1 & 92,6 & 0,086 \\
\hline
\end{tabular}

Fonte: Os autores/2017

Tabela 7. Diferença da taxa de acerto médio entre os termos.

\begin{tabular}{lcccc} 
Turmas & Acerto & IC Ins & IC Sup & Resultado \\
\hline $7^{\circ} \mathrm{A}-7^{\circ} \mathrm{C}$ & $-0,0319$ & $-0,1329$ & 0,0692 & Não significativo \\
\hline $7^{\circ} \mathrm{A}-8^{\circ} \mathrm{T}$ & 0,0793 & 0,0218 & 0,1803 & Não significativo \\
\hline $7^{\circ} \mathrm{C}-8 \mathrm{~T}$ & 0,1111 & 0,0075 & 0,2148 & Significativo \\
\hline
\end{tabular}

Fonte: Os autores/2017

desempenho de cada turma estudada, em cada seção do questionário, foi comparado e os resultados estão dispostos na Tabela 6. Observa-se que o último período do curso apresenta conhecimento significativamente superior sobre as questões referentes aos benefícios da amamentação para a mulher e ao recém-nascido. Nesse contexto, essa turma, de forma geral, apresentou menores índices de erros em comparação à turma do sétimo período $\mathrm{C}$, porém a turma $\mathrm{A}$ apresentou índice semelhante ao último período do curso (Tabela 7).

\section{DISCUSSÃO}

Este estudo permitiu identificar o perfil sociodemográfico e o conhecimento sobre aleitamento materno por alunos do curso de graduação em En- fermagem em Universidade privada do interior do estado de São Paulo, a partir da amostra de 84 alunos, os dados encontrados neste estudo são possivelmente semelhantes a de outros alunos de graduação em universidade privadas de mesmo porte.

O estudo realizado por Casagrande (13) no curso de enfermagem mostrou que apenas uma pessoa declarou ter um filho e uma pessoa respondeu ter amamentado, nossos achados, porém, diferem do estudo anterior, já que20 pessoas responderam ter filhos e 17 pessoas responderam que já amamentaram. Nesse mesmo contexto, no estudo sobre aleitamento materno feito por Badagnan ${ }^{(9)}$, apenas duas pessoas alegaram ter filhos e a média de idade dos alunos foi de 21 anos. No presente estudo, 20 alunos responderam que tinham filhos e a média de idade foi de 26,8 anos, assim, podemos observar pessoas mais velhas, o que justifica, em parte, maior número de pessoas com filhos. Tais divergências demonstram diferença sociodemográfica entre regiões do país entre estudantes universitários com e sem filhos.

Nakamura et al $^{(14)}$ realizaram um estudo com 346 meninas de uma escola de alto nível social (escola A - 149 alunas), e uma escola de baixo nível social (escola B - 197 alunas), com idade entre 9 a 15 anos, que responderam um questionário sobre aleitamento materno. Em relação a "O que você acha do leite de peito para o bebê?", 45 meninas (30\%) da escola A e 105 meninas $(54 \%)$ da escola $B$ responderam que "Protege o bebe", o que se considera uma baixa porcentagem sobre esse conceito, comparando com o nosso estudo no qual 84 alunos (100\%) responderam que o leite materno protege o bebê. No estudo citado, uma segunda pergunta feita para as meninas foi "O que você acha da mãe dar de mamar no peito para o bebê?", e 116 (78\%) alunas da escola A e 144(73\%) alunas da escola $B$ responderam "Aproxima a mãe do bebê", já no presente estudo, $100 \%$ dos alunos responderam que aumentam o vínculo da mãe e do bebê. Dessa forma, conclui-se que ainda falta um pouco do conscientização das meninas das escolas sobre o conceito de que o aleitamento materno aproxima a mãe do bebê.

Com relação ao desempenho dos participantes sobre o conhecimento em aleitamento materno, era esperado que os alunos de enfermagem do último período obtivessem melhor desempenho, considerando a diferença dos três anos já cursados na graduação. Isso foi observado em relação a uma das turmas (sétimo $C$ ), porém não em relação ao sétimo período $A$, o qual obteve semelhante ao oitavo período. Em estudos brasileiros realizados por Arantes $^{(8)}$ e Caldeira ${ }^{(15)}$ com profissionais de equipes de saúde da família os 
dados se corroboram no desempenho entre os profissionais que se tornaram 66 enfermeiros.

O estudo do autor Lelis( ${ }^{(16)}$ mostra que vários fatores evidenciam a baixa frequência na prática do aleitamento materno, seja pela dificuldade do acesso da mãe aos serviços especializados, seja por ela não saber o modo correto de como amamentar após a alta hospitalar. Nosso estudo, porém, demonstrou que, dentre as pessoas com filhos $(n=20)$ (todas as respostas foram de mulheres), 17 delas relataram ter amamentado, o que demonstra alta porcentagem $(85 \%)$, demonstrando um bom conhecimento e acesso da população local à informação e assistência sobre o aleitamento materno.

No geral, neste estudo constatou-se uma diferença importante entre conhecimentos sobre o aleitamento, quando se comparou aos sétimos e oitavo termos, possibilitando identificar necessidade de melhorar o preparo destes estudantes durante a graduação para o aconselhamento materno. Seria interessante, além da avaliação de conhecimentos, investigar por meio de pesquisa qualitativa, com grupos focais, a percepção dos estudantes sobre os aspectos que eles precisam aprender, com o intuito de planejar a inserção de ensino teórico-prático no currículo não só do curso de enfermagem, mas em outros cursos da área da saúde, e assim gerar maior conhecimento e habilidades.

\section{CONCLUSÃO}

Neste estudo, avaliamos o conhecimento dos alunos de enfermagem sobre o aleitamento materno, e exploramos os aspectos sociodemográficos dos alunos de graduação em enfermagem e, apesar do conhecimento sobre as vantagens do aleitamento, evidenciou-se conhecimento inferior ao esperado no que se refere ao processo fisiológico do aleitamento materno. 2

\section{Referências}

1. Morceli G, França EL, Magalhães VB, Damasceno DC, Calderon IM, Honorio-França $\mathrm{AC}$. Diabetes induced immunological and biochemical changes in human colostrum. Acta Paediatr 2011; 100(4): 550-6. DOI: 10.1111/j. 1651-2227.2010.02070.x.

2. Moraes LL; Campos LA; Ferrari DVJ et al. Impacto da idade materna na acidez do colostro de nutrizes em maternidade do interior do Estado de São Paulo. Journal Of Human Growth And Development. 2019 29(2):153-160

3. Costa EFG, Alves VH, Souza RMP, et al. Atuação do enfermeiro no manejo clínico da amamentação: estratégias para o aleitamento materno. Rev Fund Care Online. 2018; 10(1): 217-223. DOl: http://dx.doi.org/10.9789/21755361.2018.v10i1.217-223

4. Abdala MAP. Aleitamento Materno como programa de ação de saúde preventiva no Programa de Saúde da Família. Universidade Federal de Minas Gerais. Faculdade de medicina. Núcleo de Educação em saúde coletiva, Uberaba, 10 Set 2016. Disponível em: /www.nescon.medicina.ufmg.br/biblioteca/imagem/3141.pdf.

5. Chaves RG, Lamounier JA, César CC. Factors associated with duration of breastfeeding. J Pediatr 2007; 83(3): 241-246. DOI 10.2223/JPED.1610. 6 . World Health Organization. Obesity: preventing and managing the global epidemic. Report of a WHO consultation, World Health Organization Technical Report Series. Geneva, Switzerland:World Health Organization; 2000. 7. Capucho LB, Lima RCD, Massaroni L, Primo CC. Fatores que interferem na amamentação exclusive. Rev Bras. Pesq. Saude. 2017; 19(1): 108-113.

8. Arantes CIS, Montrone AVG, Milioni DB. Concepções e conhecimento sobre amamentação de profissionais da atenção básica à saúde. Revista Eletrônica de Enfermagem. 2008; 10: 4-6.

9. Badagnan, H.F; Oliveira HS. Conhecimento de estudantes de um curso de
Enfermagem sobre aleitamento materno. Acta Paul Enferm. 2012; 25(5): 708-712.

10. Muniz, M.D. Benefícios do aleitamento materno para a puérpera e o neonato: atuação da equipe de saúde da família. Universidade Federal de Minas Grais. Faculdade de medicina Núcleo de Educação em Saúde coletiva, 10 Set 2016. Disponível em: www.nescon.medicina.ufmg.br/biblioteca/ imagem/2843.pdf.

11. Ministério da Saúde. Política Nacional de Alimentação e Nutrição 1. Ed. Brasília, DF: Editora MS; 2013/2014, 1(1) 28.

12. Venancio SI, Escuder MML, Saldiva SRDM, Giugliani ERJ. A prática do aleitamento materno nas capitais brasileiras e Distrito Federal: situação atual e avanços. J. Pediatr. 2010; 86(4):317-324.

13. Casagrande AA. Avaliação do conhecimento sobre aleitamento materno entre os alunos do último período dos cursos da área da saúde na Universidade Federal de Santa Catarina. (Trabalho de conclusão de curso) - Universidade Federal de Santa Catarina, Florianópolis, 26 Jun 2016.

14. Nakamura S. Percepção e conhecimento de meninas escolares sobre 0 aleitamento materno. Jornal de Pediatria - 2003; 79(2).

15. Caldeira AP, Aguiar GN, Magalhães WAC, Fagundes GC. Conhecimentos e práticas de promoção do aleitamento materno em Equipes de Saúde da Família em Montes Claros, Minas Gerais, Brasil. Cadernos de Saúde Pública, 2007.

16. Lelis L.S.C. Aleitamento materno exclusivo a criança até os seis meses de idade: avanços e desafios. Universidade Federal de Minas Gerais. Faculdade de Medicina. Núcleo de Educação em Saúde Coletiva. Conselheiro Lafaiete, 22 Agost 2016. Disponível em: www.nescon.medicina.ufmg.br/bibliotecal imagem/3401.pdf. 